EPJ Web of Conferences 81, 01024 (2014)

DOI: $10.1051 /$ epjconf/ 20148101024

(C) Owned by the authors, published by EDP Sciences, 2014

\title{
Recent ATLAS results in the field of meson physics
}

\author{
Rui Wang ${ }^{1, a}$ \\ on behalf of the ATLAS Collaboration \\ ${ }^{1}$ Department of Physics and Astronomy, University of New Mexico, \\ 1919 Lomas Blvd NE, Albuquerque, NM, US, 87131
}

\begin{abstract}
Recent results on flavor physics are presented, using data from the ATLAS detector. The $\Upsilon(n S)$ and charmonium inclusive production cross sections are discussed. $J / \psi$ production associated with a $W$ vector boson is presented. An inclusive analysis of $B^{+}$meson production is shown. Results on the production of heavy quarkonia are complemented by the measurement of inclusive $\phi$ meson production. The latest information on heavy flavor spectroscopy and searches for new physics using $B$-meson rare decays is also covered. These results are based on data samples collected during the 2011 and 2012 LHC running periods.
\end{abstract}

\section{The $\phi(1020)$ production cross section measurement}

As a theory of the physics at high momentum transfer, perturbative quantum chromodynamics (pQCD) is very successful. However, for interactions at low momentum transfers, there is no single best model. The $\phi(1020)$ meson is produced in hard scatters during $p p$ interactions as well as in hadronisation. Therefore, accurate measurements of $\phi(1020)$ meson production can be used to tune phenomenological fragmentation models.

The $\phi(1020)$ meson production is measured using $383 \mu \mathrm{b}^{-1}$ of $7 \mathrm{TeV}$ data collected in 2010 [1] using the ATLAS detector [2]. The $\phi(1020)$ is reconstructed using kaons identified by their energy loss in the pixel detector. The kaons are required to have transverse momentum $p_{\mathrm{T}}>230 \mathrm{MeV}$ and total momentum $p<800 \mathrm{MeV}$. The analysis is restricted to the fiducial region $500<p_{\mathrm{T}}(\phi)<1200 \mathrm{MeV}$ and $|y(\phi)|<0.8$, where $y$ is rapidity. The integrated production cross section for $\phi(1020) \rightarrow K^{+} K^{-}$is found to be $570 \pm 8$ (stat. $) \pm 66$ (sys.) \pm 20 (lumi.) $\mu$ b. The differential cross sections are shown in Fig. 1 along with comparisons to various Monte Carlo (MC) based predictions. The EPOS-LHC and PYTHIA6 DW tunes give the best agreement.

\section{$2 W$ plus $J / \psi$ associated production}

The measurement of $W$ plus $J / \psi$ associated production can be used to test the relative contributions of the color singlet (CS) and color octet (CO) processes to $Q \bar{Q}$ production.

\footnotetext{
a e-mail: Rui.Wang@cern.ch
} 
EPJ Web of Conferences
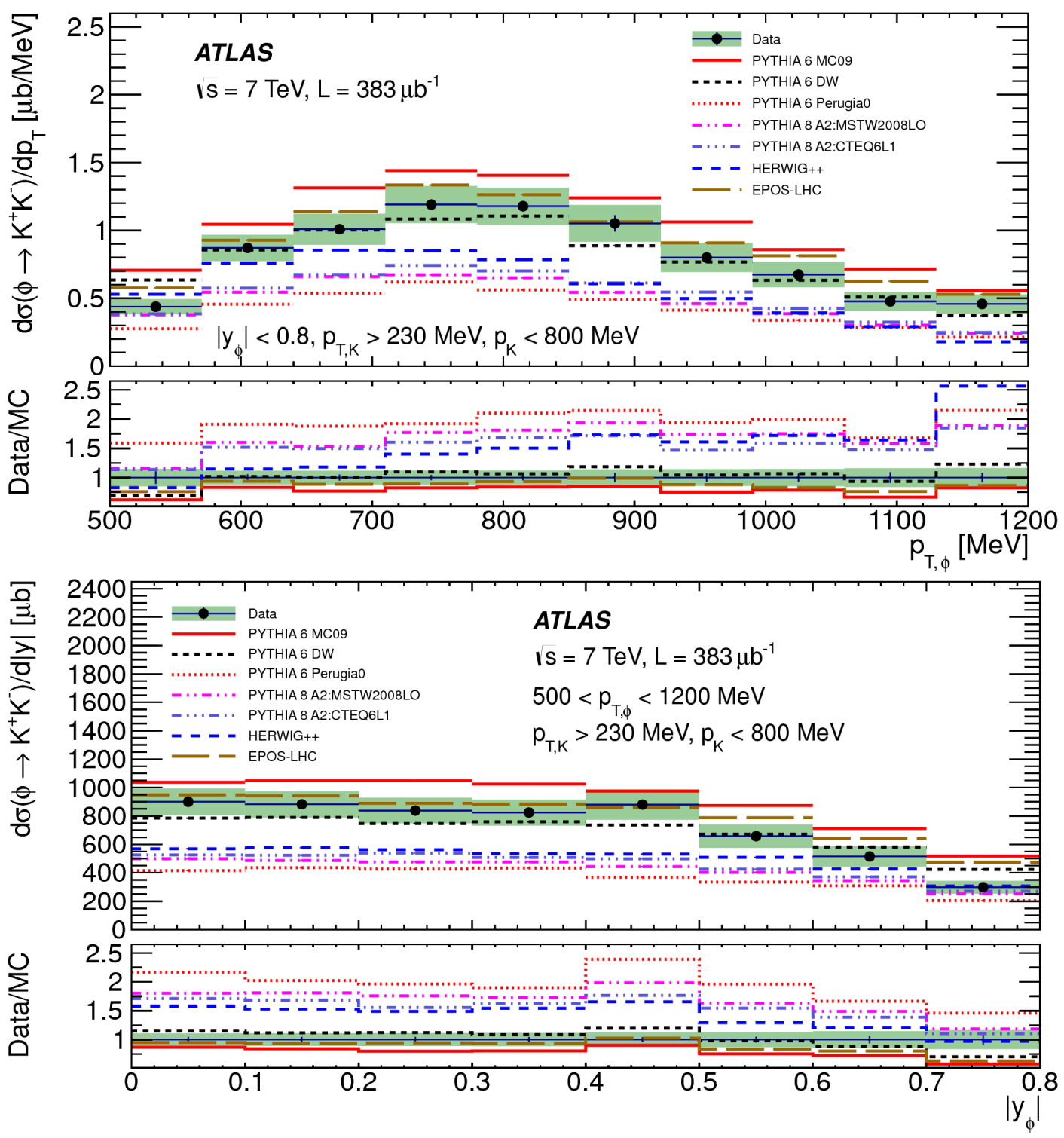

Figure 1: The $\phi(1020) \rightarrow K^{+} K^{-}$cross section as a function of $p_{\mathrm{T}}(\phi)$ (left) and $|y(\phi)|$ (right), in the fiducial region of $500<p_{\mathrm{T}}(\phi)<1200 \mathrm{MeV}$ and $|y(\phi)|<0.8$.

The measurement is based on $4.5 \mathrm{fb}^{-1}$ of $7 \mathrm{TeV}$ pp collision data collected by ATLAS in 2011 [3]. In total, $29.2_{-6.5}^{+7.5}$ prompt $J / \psi$ plus $W$ boson events are observed, corresponding to a signal $5.1 \sigma$ significance.

As shown in Fig. 2, left, these $W$ boson plus prompt $J / \psi$ events include both single parton scattering (SPS) events and double parton scattering (DPS) events. The DPS-subtracted rate appears to 
MESON $2014-13^{\text {th }}$ International Workshop on Production, Properties and Interaction of Mesons
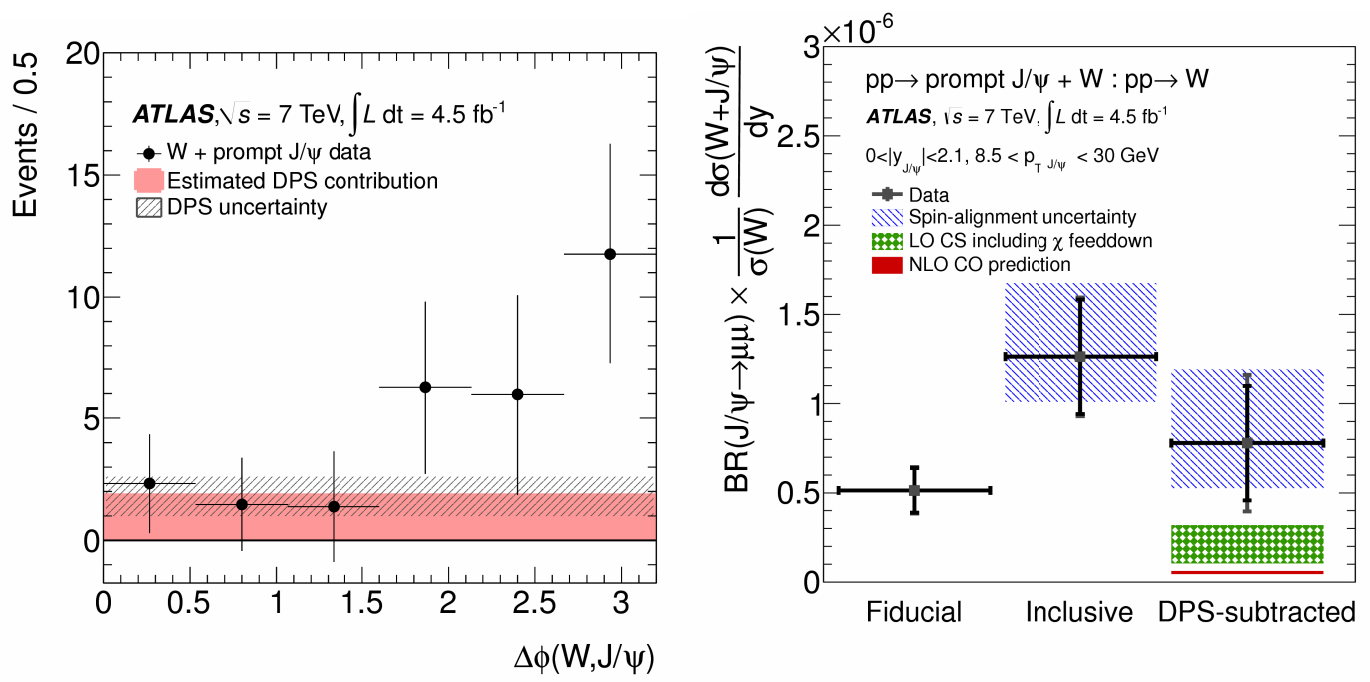

Figure 2: The sPlot-weighted azimuthal angle between the $W$ and the $J / \psi$ with a flat DPS contribution from MC overlaid (left). The $W$ plus the $J / \psi$ production cross-section ratio in the $J / \psi$ fiducial region (Fiducial), after correction for $J / \psi$ acceptance (Inclusive), and after subtraction of the double parton scattering component (DPS-subtracted) (right).

be dominated by CS (Fig. 2, right). However, both of the leading order (LO) CS and next-to-leading order (NLO) CO predictions are compatible with the measured result at the $2 \sigma$ level.

\section{Measurement of the $\chi_{c 1,2}$ and $\psi(2 S)$ production cross sections}

Together with the $J / \psi$ production cross section, $\chi_{c}$ and $\psi(2 S)$ production cross section measurements can give a precise picture of the production of both prompt and non-prompt charmonium below the
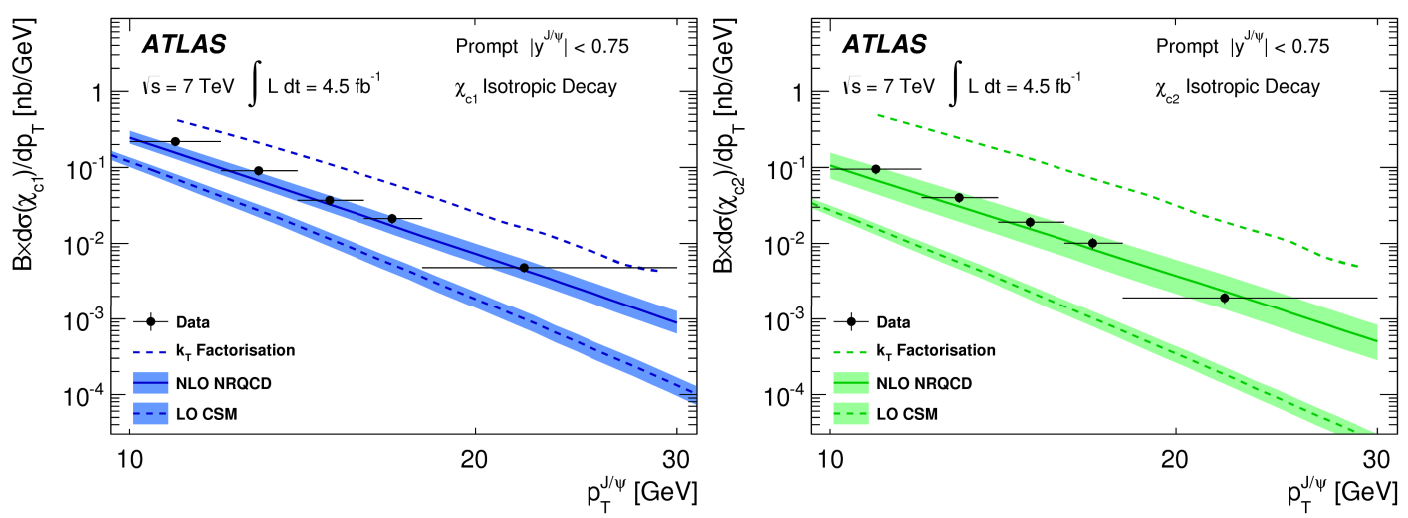

Figure 3: Differential cross-sections for prompt $\chi_{c 1}$ (left) and $\chi_{c 2}$ (right) production. 

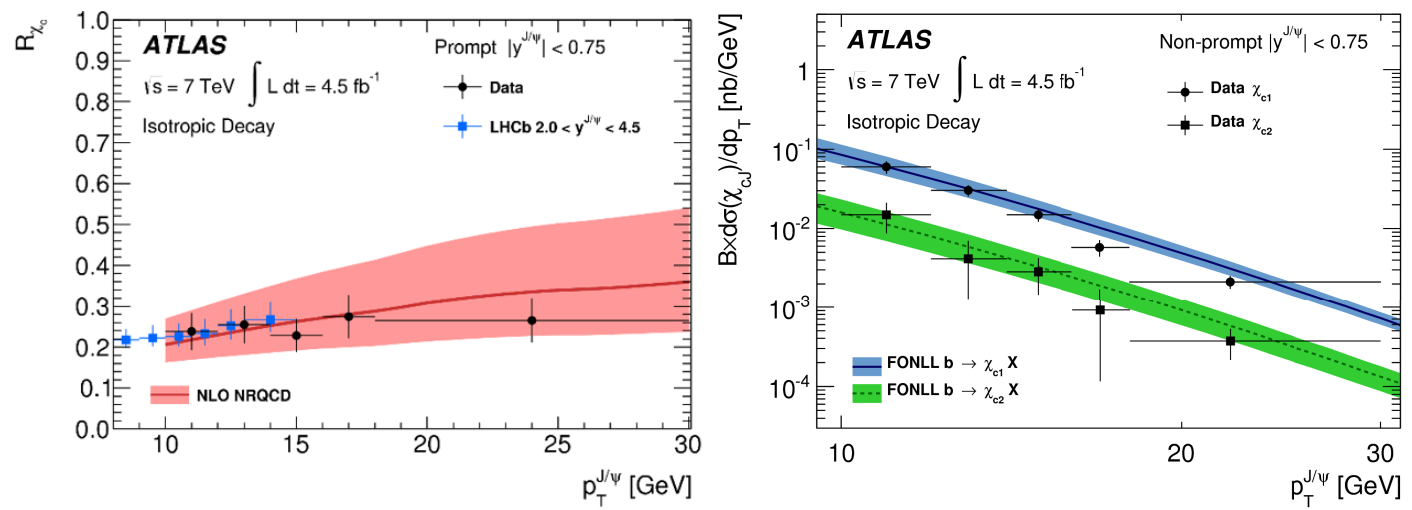

Figure 4: The fraction, $R_{\chi_{c}}$, of prompt $J / \psi$ events produced in $\chi_{c}$ decays is shown as a function of $p_{\mathrm{T}}^{J / \psi}$ (left). Differential cross-sections for non-prompt $\chi_{c 1}, \chi_{c 2}$ (right).

$D \bar{D}$ threshold. The prompt production normally includes both direct production from $p p$ collisions and production from decays of heavier charmonium states (the feed-down contribution). However, since $\psi(2 S)$ is the highest state below the $D \bar{D}$ threshold, it has no contribution from higher states. The non-prompt production is contributed by the $b$ hadron decays.

The $\chi_{c 1,2} \rightarrow J / \psi \gamma$ production cross section is measured using $4.5 \mathrm{fb}^{-1}$ of $7 \mathrm{TeV} 2011$ data in the ranges $10<p_{\mathrm{T}}(J / \psi)<30 \mathrm{GeV}$ and $|y(J / \psi)|<0.75$ [4]. The $\psi(2 S) \rightarrow J / \psi \pi \pi$ production cross section is measured using $2.1 \mathrm{fb}^{-1}$ of $7 \mathrm{TeV} 2011$ data in the ranges $10<p_{\mathrm{T}}<100 \mathrm{GeV}$ and $|y|<$ 2.0 [5]. The prompt production data are compared to different models (Fig. 3 and Fig. 5, left). NLO non-relativistic QCD (NRQCD) is compatible in both cases. The LO Color Singlet Model (CSM) underestimates the $\chi_{c 1,2}$ production cross section and the $\psi(2 S)$ production cross section. The $k_{\mathrm{T}}$ factorisation approach overestimates the $\chi_{c 1,2}$ production cross section and underestimates the $\psi(2 S)$
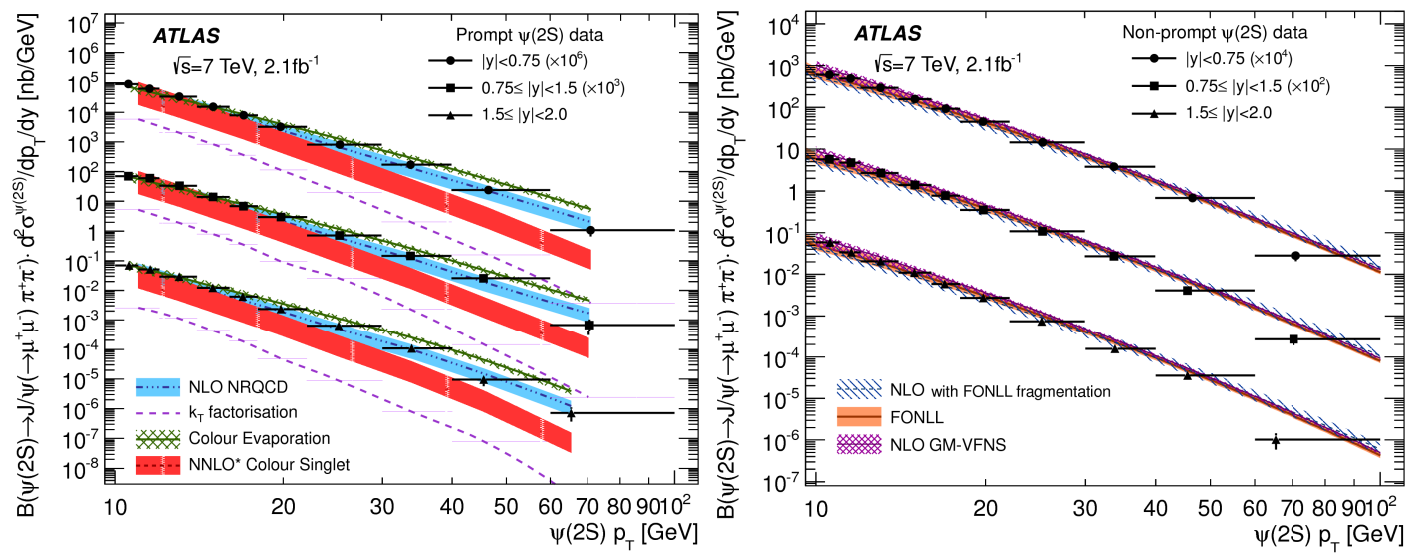

Figure 5: Differential cross-sections for prompt $\psi(2 S)$ (left) and non-prompt $\psi(2 S)$ (right) production. 
production cross section. These suggest that higher-order corrections or $\mathrm{CO}$ contributions to the cross sections not included in either prediction may be important. Between $20 \%$ and $30 \%$ of prompt $J / \psi$ mesons are produced in $\chi_{c 1}$ feed-down (Fig. 4, left). As is shown in Fig. 4, right and Fig. 5, right, the non-prompt production data are compatible with the FONLL predictions.

\section{Measurement of the $\Upsilon(n P)$ production cross section}

As a complement to charmonium system studies, the $\Upsilon$ family allows more dependable theoretical calculations due to its members' larger masses. Additionally, the impact of spin-alignment uncertainties is mitigated. Like charmonium, $\Upsilon$ mesons are produced directly in $p p$ collisions or from decay of excited states.

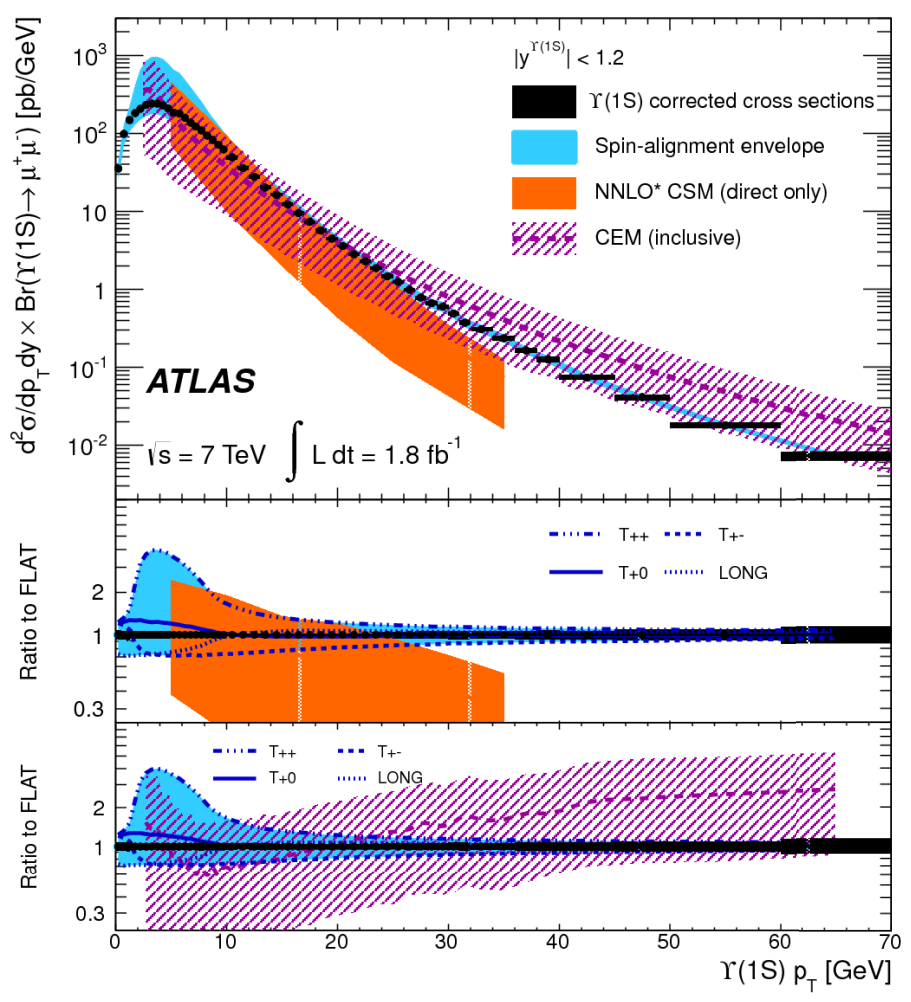

Figure 6: The $\Upsilon(1 S)$ differential cross sections multiplied by the di-muon branching fraction, $d^{2} / d p_{\mathrm{T}} d y \times \operatorname{Br}\left(\Upsilon \rightarrow \mu^{+} \mu^{-}\right)$.

The $\Upsilon(n S) \rightarrow \mu^{+} \mu^{-}$production cross sections has been measured using $1.8 \mathrm{fb}^{-1}$ of $7 \mathrm{TeV} 2011$ data collected by ATLAS in the ranges $p_{\mathrm{T}}<70 \mathrm{GeV}$ and $|y|<2.25$ [6]. The integrated cross sections are found to be $\Upsilon(1 S)=8.01 \pm 0.02$ (stat. $) \pm 0.36$ (sys.) \pm 0.31 (lumi. $) \mathrm{nb}, \Upsilon(2 S)=$ $2.05 \pm 0.01$ (stat.) \pm 0.12 (sys.) \pm 0.08 (lumi.) $\mathrm{nb}$, and $\Upsilon(3 S)=0.92 \pm 0.01$ (stat. $) \pm 0.07$ (sys.) \pm 0.04 (lumi.) nb. The differential cross sections (Fig. 6) can be well described by the CS model in the low $p_{\mathrm{T}}$ region but are underestimated in the high $p_{\mathrm{T}}$ region. The feed-down contribution has not been included into the CSM calculations. The color evaporation model (CEM) gives a better description, it includes the 
EPJ Web of Conferences

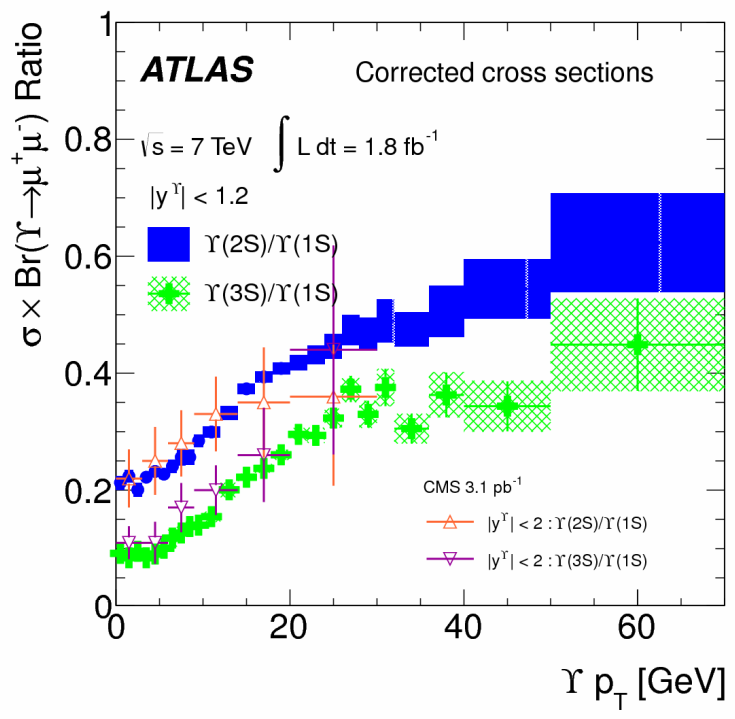

Figure 7: The ratios of differential $\Upsilon(2 S) / \Upsilon(1 S)$ and $\Upsilon(3 S) / \Upsilon(1 S)$ cross sections multiplied by the di-muon branching fractions versus $\Upsilon p_{\mathrm{T}}$ in the central rapidity regions.

feed-down naturally. In Fig. 7, the increase in the $p_{\mathrm{T}}$ range of $5 \mathrm{GeV}$ to $30 \mathrm{GeV}$ may be related to the feed-down contribution.

\section{Observation of a new $\chi_{b}$ state}

$\chi_{b}(n P)$ states are sought using $4.9 \mathrm{fb}^{-1}$ of ATLAS $20117 \mathrm{TeV}$ data [7], through decay modes of $\chi_{b}(n P) \rightarrow \Upsilon(1 S, 2 S) \gamma$. In this measurement, a di-muon sample with requirments of $p_{\mathrm{T}}>4 \mathrm{GeV}$ and
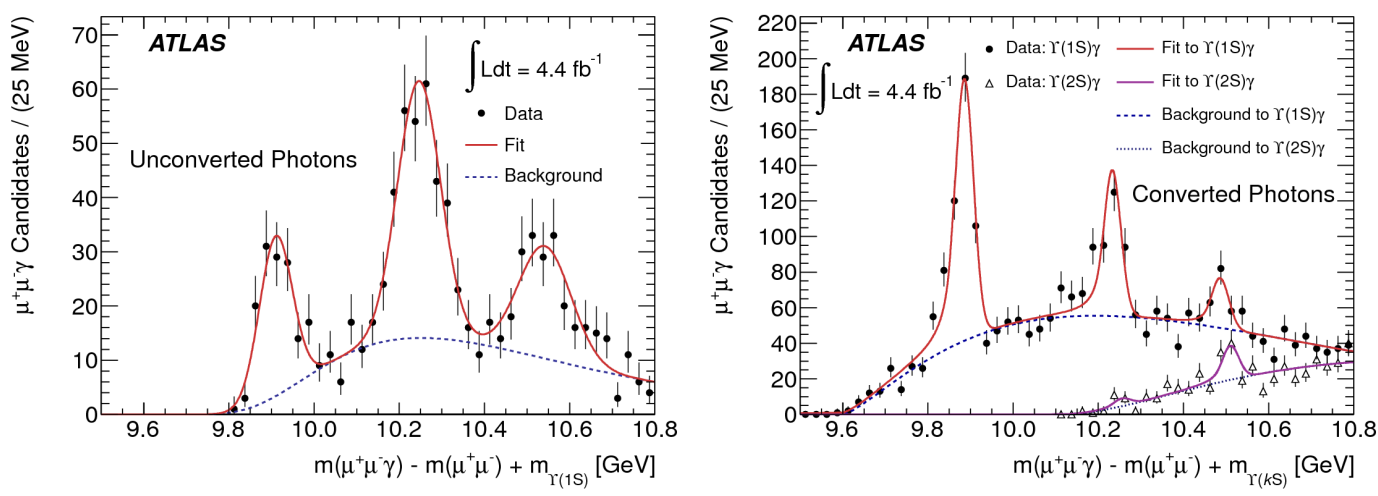

Figure 8: The mass distribution of $\chi_{b} \rightarrow \Upsilon(1 S) \gamma$ candidates for unconverted photons (left) and the mass distributions of $\chi_{b} \rightarrow \Upsilon(k S) \gamma(\mathrm{k}=1,2)$ candidates formed using converted photons (right). 
pseudorapidity $|\eta|<2.3$ for each muon has been used for $\Upsilon(1 S, 2 S)$ candidate selection. A photon is combined with each $\Upsilon$ candidate. Converted photons reconstructed from $e^{+} e^{-}$track pairs in the Inner Detector (ID) and unconverted photons reconstructed from electromagnetic calorimeter energy deposits are used.

As shown in the mass difference $\left(m\left(\mu^{+} \mu^{-} \gamma\right)-m\left(\mu^{+} \mu^{-}\right)+m_{P D G}(\Upsilon)\right)$ distributions (Fig. 8), a new structure centered at mass $10.530 \pm 0.005$ (stat.) \pm 0.009 (syst.) $\mathrm{GeV}$ is observed with a significance of more than $6 \sigma$ in each of the unconverted and converted photon selections. This is interpreted as the $\chi_{b}(3 P)$ state.

\section{Angular analysis of $B_{d} \rightarrow K^{* 0}\left(K^{+} \pi^{-}\right) \mu^{+} \mu^{-}$events}

The angular distributions of the $B_{d} \rightarrow K^{* 0}\left(K^{+} \pi^{-}\right) \mu^{+} \mu^{-}$four particle final state are sensitive to physics beyond the Standard Model (SM). Two important observables are the forward-backward asymmetry of the muons, $A_{F B}$, and the longitudinal polarization fraction of the $K^{* 0}, F_{L}$. They can be extracted from the decay width which depends on the angle between those two muon momenta, the angle between the kaon and pion momenta, and the momentum transfer $q^{2}=m^{2}\left(\mu^{+} \mu^{-}\right)$. The measurement uses 4.9 $\mathrm{fb}^{-1}$ of $7 \mathrm{TeV} 2011$ data recorded by ATLAS [8]. As shown in Fig. 9, both $A_{F B}$ and $F_{L}$ as measured by ATLAS are compatible with SM predictions and with other measurements.
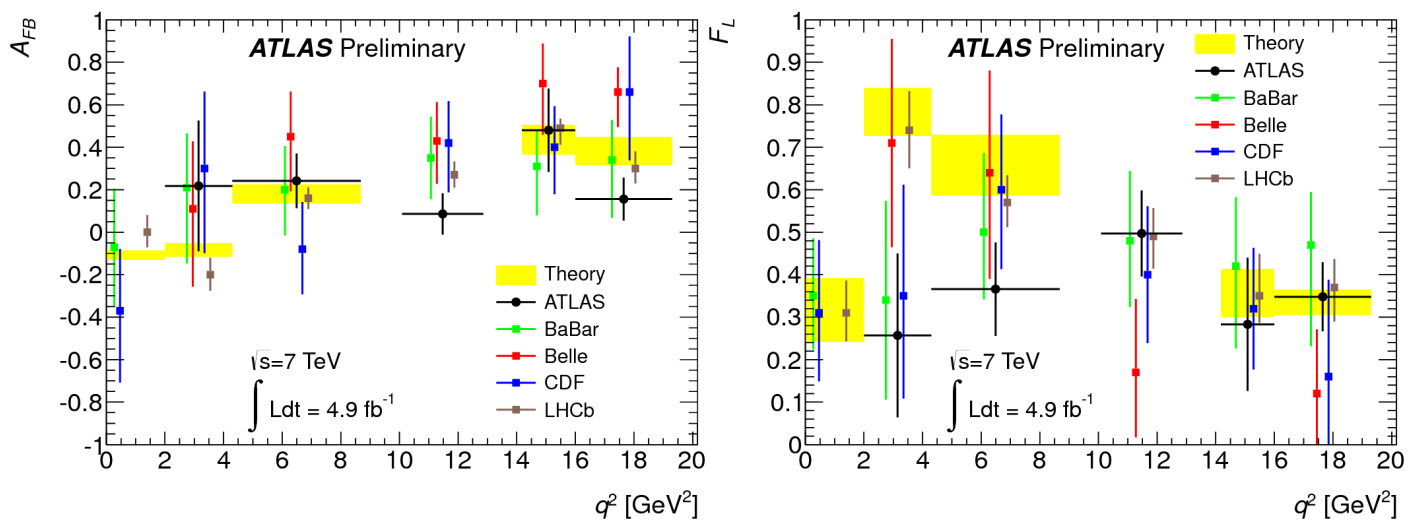

Figure 9: $A_{F B}$ (left) and $F_{L}$ (right) as a function of momentum transfer $q^{2}$. The SM theoretical predictions have been calculated for the limit of small vector meson energy and for the limit of large vector meson energy. No expectation is given for the central $q^{2}$ region. The $q^{2}$ bins corresponding to $J / \psi$ and $\psi(2 S)$ resonances are not filled.

\section{Angular analysis of $B_{s} \rightarrow J / \psi \phi$ enents}

The $B_{s} \rightarrow J / \psi \phi$ process is also sensitive to new physics beyond the SM. CP violation in this decay occurs due to interference between direct decays and decays occurring through $B_{s}^{0}-\bar{B}_{s}^{0}$ mixing. The $\mathrm{CP}$ states are separated statistically through the time dependence of the decay and angular correlations among the final state particles. With $4.9 \mathrm{fb}^{-1}$ of $7 \mathrm{TeV} 2011$ data, the ATLAS measurement of the CP violating weak phase is $\phi_{s}=0.12 \pm 0.25$ (stat.) \pm 0.05 (syst.) rad. The ATLAS measurement of the difference of widths of the heavy and light mass eigenstates is $\Delta \Gamma_{s}=0.053 \pm 0.021$ (stat.) \pm 0.010 (syst.) $\mathrm{ps}^{-1}$ [9]. Both are compatible with the SM predictions (Fig. 10). 


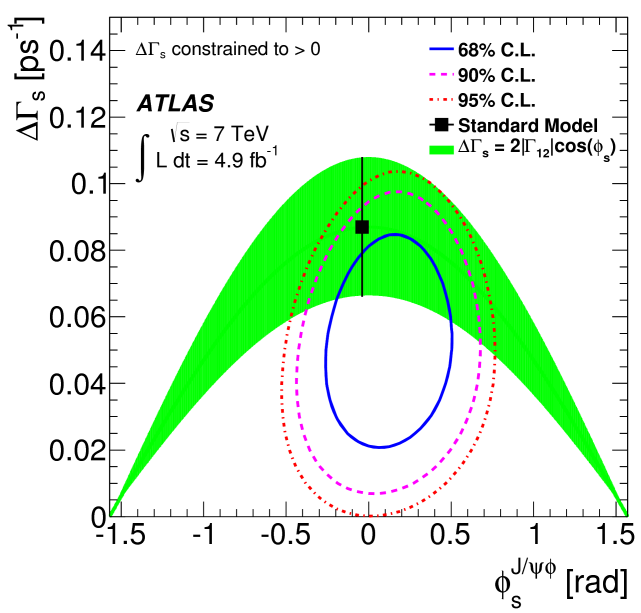

Figure 10: Likelihood contours in the $\phi_{s}-\Delta \Gamma_{s}$ plane. The green band is the theoretical prediction of mixing-induced $\mathrm{CP}$ violation.

\section{Search for the rare decay $B_{s}^{0} \rightarrow \mu^{+} \mu^{-}$}

Because flavor changing neutral currents (FCNC) are highly suppressed in the SM, the predicted branching ratio of $B_{s}^{0} \rightarrow \mu \mu$ is quite small. It may be enhanced or reduced by new physics processes. At ATLAS, the decay has been searched with respect to a prominent reference decay $\left(B^{+} \rightarrow J / \psi K^{+}\right)$

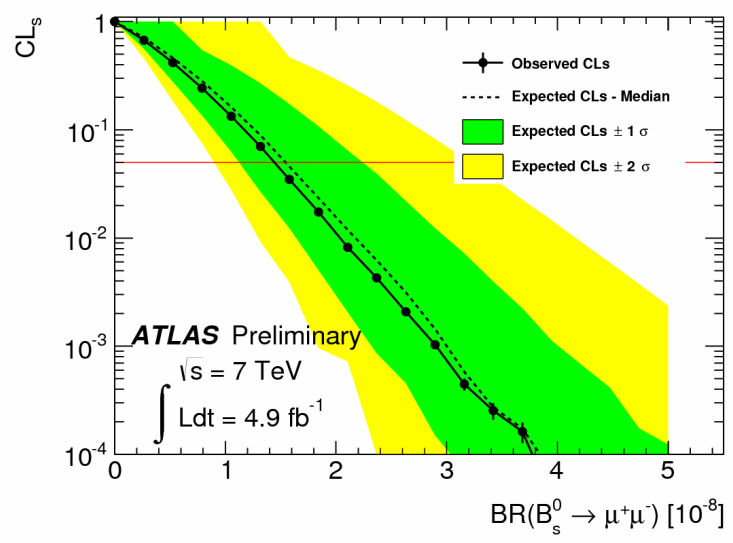

Figure 11: Observed confidence levels (CLs, circles) are shown as a function of $\operatorname{BR}\left(B_{s}^{0} \rightarrow \mu^{+} \mu^{-}\right)$. The $95 \% \mathrm{CL}$ limit is indicated by the horizontal (red) line. The green and yellow bands correspond to $\pm 1 \sigma$ and $\pm 2 \sigma$ fluctuations on the expectation (dashed line).

using $4.9 \mathrm{fb}^{-1}$ of $7 \mathrm{TeV} 2011$ data [10]. Six events in the signal region have been observed with 6.75 background events expected. Thus ATLAS sets a limit on the branching ratio at $1.5 \times 10^{-8} @ 95 \% \mathrm{CL}$ (Fig. 11). 


\section{Measurement of the $B^{+}$production cross section}

Precise measurements of $B$-hadron production cross sections in $p p$ collisions at LHC can provide tests of QCD calculations for heavy-quark production at high center-of-mass energies and in wide $p_{\mathrm{T}}$ and $y$ ranges. The $B^{+} \rightarrow J / \psi K^{+}$production cross section is measured using $2.4 \mathrm{fb}^{-1}$ of early 20117
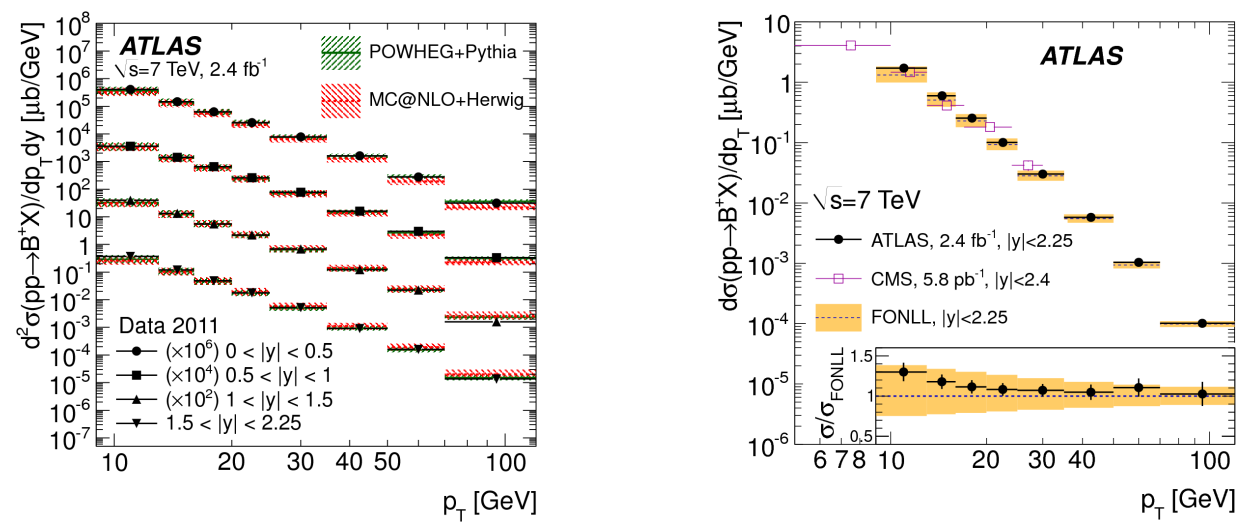

Figure 12: The doubly-differential cross-section for $B^{+}$production as a function of $p_{\mathrm{T}}$ and $y$ (left). The differential cross-section for $B^{+}$production versus $p_{\mathrm{T}}$, integrated over rapidity, compared with predictions using the FONLL calculation with a hadronization fraction $f_{b \rightarrow B^{+}}$of $(40.1 \pm 0.8) \%$ (right).

$\mathrm{TeV}$ data [11]. The differential cross section in the kinematic range $9 \mathrm{GeV}<p_{\mathrm{T}}<120 \mathrm{GeV}$ and $|y|<$ 2.25 is shown in Fig. 12. Different model predictions have been compared to the measured data, and they are compatible within theoretical uncertainties.

\section{$10 B_{c}$ meson observation}

The $B_{c}^{ \pm}$meson is a bound state of the two heaviest quarks able to form a stable state. Weak decays of

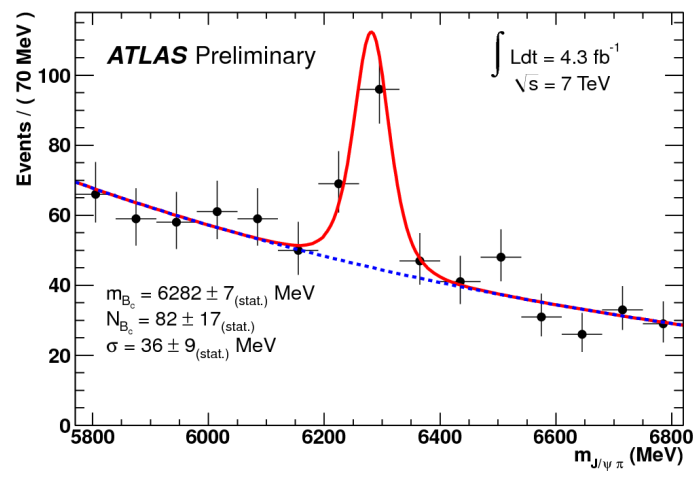

Figure 13: The invariant mass distribution of reconstructed $B_{c}^{ \pm} \rightarrow J / \psi \pi^{ \pm}$candidates. 
the $B_{c}^{ \pm}$meson provide a unique probe of heavy quark dynamics that is inaccessible to $b \bar{b}$ or $c \bar{c}$ bound states. The $B_{c}^{ \pm}$meson is reconstructed using $20117 \mathrm{TeV}$ data in the decay mode $B_{c} \rightarrow J \psi \pi$ [12]. With $4.3 \mathrm{fb}^{-1}$ data, $82 \pm 17 B_{c}$ ground state mesons have been extracted using an unbinned maximum likelihood fit (Fig. 13). The $B_{c}$ mass returned by the fit is $6282 \pm 7 \mathrm{MeV}$, in agreement with the world average value.

\section{References}

[1] ATLAS Collaboration, Eur. Phys. J. C74, 2895 (2014).

[2] ATLAS Collaboration, JINST 3, S08003 (2008).

[3] ATLAS Collaboration, JHEP 04, 172 (2014).

[4] ATLAS Collaboration, JHEP 07, 154 (2014).

[5] ATLAS Collaboration, JHEP 09, 079 (2014).

[6] ATLAS Collaboration, Phys. Rev. D 87, 052004 (2013).

[7] ATLAS Collaboration, Phys. Rev. Lett. 108, 152001 (2012).

[8] ATLAS Collaboration, ATLAS-CONF-2013-038, https://cds.cern.ch/record/1537961.

[9] ATLAS Collaboration, Phys. Rev. D 90, 052007 (2014).

[10] ATLAS Collaboration, ATLAS-CONF-2013-076, https://cds.cern.ch/record/1562934.

[11] ATLAS Collaboration, JHEP 10, 042 (2013).

[12] ATLAS Collaboration, ATLAS-CONF-2012-028, https://cds.cern.ch/record/1430737. 